SLAC-PUB-1244

$(\mathrm{T} / \mathrm{E})$

May 1973

\title{
FLUX INDEPENDENT MEASUREMENTS OF DEEP INELASTIC NEUTRINO PROCESSES ${ }^{*}$
}

J. D. Bjorken

Stanford Linear Accelerator Center, Stanford University, Stanford, California 94305

D. Cline

Physics Department, University of Wisconsin, Madison, Wisconsin 53706

A. K. Mann

Physics Department, University of Pennsylvania, Philadelphia, Pennsylvania 19104

\section{ABSTRACT}

The scaling variable $\mathrm{v}=\mathrm{xy}=2\left(\mathrm{E}_{\mu} / \mathrm{M}\right) \sin ^{2} \frac{\theta \mu}{2}$ is useful for the description of deep inelastic neutrino-nucleon scattering processes. This variable is determined solely by the momentum and angle of the out-going lepton. The normalized scattering distribution in this variable is independent of incident lepton energy and flux, provided scale-invariance is valid. The sensitivity to various hypothetical scale breaking mechanisms is discussed.

(Submitted to Phys. Rev. Comments and Addenda.)

* Supported in part by the U.S. Atomic Energy Commission. 
Experiments which attempt to measure deep inelastic neutrino-nucleon cross sections are plagued with the difficult problem of determining the neutrino flux. It is clearly desirable to find ways of extracting information from such experiments which is flux-independent. Experimental studies along these lines have already been carried out by Myatt and Perkins. ${ }^{1}$ Recently, Cline and Paschos ${ }^{2}$ have analyzed moments of the scaling variable $x=Q^{2} / 2 M \nu$, which yield information regarding current algebra sum rules, provided scaling is correct. Paschos and Zakharov ${ }^{3}$ have also put bounds on $<\mathrm{E}_{\mu} / \mathrm{E}_{\nu}>$ and $<\mathrm{Q}^{2} / 2 \mathrm{ME}_{\nu}>$ which depend only on the hypothesis of scale invariance.

Here we consider the case where the only accurately determined quantities in an experiment are the secondary muon momentum $\mathrm{E}^{\prime}$ and its production angle $\theta$. We find that some theoretical questions, in particular the validity of dimensional scaling, can be answered from this information alone. The key lies in the fact that the scale invariant quantity

$$
\mathrm{v}=\mathrm{Q}^{2} / \mathrm{s}=\mathrm{Q}^{2} / 2 \mathrm{ME}=\left(\frac{\mathrm{Q}^{2}}{2 \mathrm{M} \nu}\right)\left(\frac{\nu}{\mathrm{E}}\right)=\mathrm{xy}=\frac{2 \mathrm{E}^{\prime}}{\mathrm{M}} \sin ^{2} \frac{\theta}{2}
$$

is an observable. The normalized v-distribution $\frac{1}{\mathrm{~N}} \frac{\mathrm{dN}}{\mathrm{dv}}$ should then be scale invariant (i. e. independent of neutrino energy E) provided the deep inelastic structure functions are scale invariant. Furthermore, interesting information about the structure functions can be obtained from the shape of the v-distribution and, in particular, its moments.

\section{Tests of Scaling}

The cross section for the process $\nu_{\mu}+\mathrm{N} \rightarrow \mu^{-}+$(hadrons) may be 
written $^{5}$ (for monoenergetic neutrinos with $\mathrm{E} \gg 1 \mathrm{GeV}$ )

$$
\frac{\mathrm{d} \sigma}{\mathrm{dxdy}}=\frac{\mathrm{G}^{2} \mathrm{~S}}{2 \pi} \mathrm{F}_{2}\left(\mathrm{x}, \mathrm{Q}^{2}\right)\left[\mathrm{L}+(1-\mathrm{y}) \mathrm{S}+(1-\mathrm{y})^{2} \mathrm{R}\right]
$$

where

$$
\begin{aligned}
& \mathrm{x}=\frac{\mathrm{Q}^{2}}{2 \mathrm{M} \nu}, \mathrm{y}=\frac{\nu}{\mathrm{E}}, \quad \mathrm{s}=2 \mathrm{mE}, \quad \nu=\mathrm{E}-\mathrm{E}^{\prime}, \quad \mathrm{Q}^{2}=4 \mathrm{EE}^{\prime} \sin ^{2} \frac{\theta}{2} \\
& \mathrm{~L}=\frac{\sigma_{\mathrm{L}}}{\sigma_{\mathrm{L}}+\sigma_{\mathrm{R}}+2 \sigma_{\mathrm{S}}}, \quad \mathrm{R}=\frac{\sigma_{\mathrm{R}}}{\sigma_{\mathrm{R}}+\sigma_{\mathrm{L}}+2 \sigma_{\mathrm{S}}}, \quad \mathrm{S}=\frac{2 \sigma_{\mathrm{S}}}{\sigma_{\mathrm{L}}+\sigma_{\mathrm{R}}+2 \sigma_{\mathrm{S}}}
\end{aligned}
$$

and we approximate $\nu^{2} \gg \mathrm{Q}^{2}$.

Let us for the moment assume that $\mathrm{F}_{2}\left(\mathrm{x}, \mathrm{Q}^{2}\right)$ scales, i. e. for $\mathrm{Q}^{2} \gtrsim 1 \mathrm{GeV}{ }^{2}$, $\mathrm{F}_{2}\left(\mathrm{x}, \mathrm{Q}^{2}\right) \approx \mathrm{F}_{2}(\mathrm{x})$, and that this is also true for $\mathrm{L}, \mathrm{R}$ and $\mathrm{S}$. An elementary calculation then reveals that

$$
\frac{1}{N} \frac{d N}{d v}=\frac{1}{\sigma_{\text {tot }}} \frac{d \sigma}{d v}=\frac{J_{v} \frac{d x}{x} F_{2}(x)\left[L+\left(1-\frac{v}{x}\right) S+\left(1-\frac{v}{x}\right)^{2} R\right]}{\int_{0}^{1} d x F_{2}(x)\left[L+\frac{1}{2} S+\frac{1}{3} R\right]} .
$$

Thus, scaling indeed implies that $\frac{1}{\mathrm{~N}} \mathrm{dN} / \mathrm{dv}$ is independent of neutrino energy $\mathrm{E}$, as anticipated. We, therefore, may fold Eq. (4) over a neutrino energy spectrum with no change in the result. For orientation we may suppose, as indicated by experiment, that $\mathrm{L} \gg \mathrm{R}, \mathrm{S}$ (unless, perhaps, $\mathrm{x} \ll 1$ ). ${ }^{6,7}$ In this case

$$
\frac{1}{N} \frac{d N}{d v} \approx \frac{\int_{v}^{1} \frac{d x}{x} F_{2}(x)}{\int_{0}^{1} d x F_{2}(x)}
$$


and with very accurate data the shape of $F_{2}(x)$ might be extracted.

Now let us suppose that scaling is violated (and that we can again neglect $R$ and S) and parametrize the violation by writing either

$$
\mathrm{F}_{2}\left(\mathrm{x}, \mathrm{Q}^{2}\right) \approx \frac{\mathrm{F}_{2}(\mathrm{x})}{\left[1+\mathrm{v} \frac{\mathrm{s}}{\Lambda^{2}}\right]^{2}}
$$

or

$$
\mathrm{F}_{2}\left(\mathrm{x}, \mathrm{Q}^{2}\right) \approx \mathrm{F}_{2}(\mathrm{x})\left[1-\epsilon \log \frac{\mathrm{Q}^{2}}{\mathrm{M}_{\mathrm{p}}^{2}}\right]
$$

The first form would arise from parton structure or finite intermediate boson mass. ${ }^{5}$ The second is the kind of breakdown expected from studies of perturbation theory in renormalizable field theories or from small anomalous dimensions in a theory scale invariant at short distances. Inserting these into Eq. (5) gives (for a nearly monochromatic beam of neutrinos)

$$
\frac{1}{N} \frac{d N}{d v}=\frac{\frac{1}{\left[1+v \frac{s>}{\Lambda^{2}}\right]^{2}} \int_{v}^{1} \frac{d x}{x} F_{2}(x)}{\int_{0}^{1} \frac{d x F_{2}(x)}{\left[1+x \frac{<s>}{2}\right]}} .
$$

For the logarithmic violation

$$
\frac{1}{N} \frac{d N}{d v}=\frac{\int_{v}^{1} \frac{d x}{x} F_{2}(x)\left[1-\epsilon\left(<\log \frac{s}{M^{2}}>+v\right)\right]}{\int_{0}^{1} d x F_{2}(x)\left[1-\epsilon\left(<\log \frac{s}{M^{2}}>+\log x-1\right)\right]}
$$


where

$$
\langle s\rangle=\frac{\int \mathrm{dEm}(\mathrm{E})(2 \mathrm{mE})}{\int \mathrm{dEm}(\mathrm{E})} \text {, etc. }
$$

with $m(E)$ given by the neutrino spectrum.

We obtain, as would be expected, either linear or logarithmic deviations from scaling behavior of the $\mathrm{v}$ distribution, with the largest effect nearest $v=1$. (See Fig. 2.) We have plotted $\frac{1}{N} \frac{d N}{d v}$ in Fig. 1, assuming $F_{2}(x)$ has the same shape as the structure function $\nu \mathrm{W}_{2}$ measured in electroproduction. ${ }^{8}$ Evidently, if $s \gg \Lambda^{2}$ this distribution could be narrowed appreciably by a scaling breakdown. Consequently, comparison of the normalized $\mathrm{v}$ distributions measured at several widely different neutrino energies, e.g., at $\left\langle\mathrm{E}_{\nu}>\approx 3\right.$, 30 and $150 \mathrm{GeV}$, will comprise a sensitive test of scaling behavior. An interesting test can be made at any neutrino energy by comparison of the observed normalized $\mathrm{v}$ distribution with that in Fig. 1. In Fig. 1 is also presented the approximate form of $\frac{1}{\mathrm{~N}} \frac{\mathrm{dN}}{\mathrm{dV}}$ for the example of $\Lambda=\mathrm{M}_{\mathrm{W}}=5 \mathrm{GeV}$ for $<\mathrm{s}>=$ $60 \mathrm{GeV}^{2}$ (a value appropriate to NAL). More generally, the modification to the scaling behavior given by (8) is displayed in Fig. 2 . It is quite insensitive to the detailed behavior of $F(x)$.

\section{Neutrino-Antineutrino Comparisons}

For a target composed of equal numbers of neutrons and protons, and neglecting $\Delta S=1$ processes, the $\mathrm{v}$ distribution for incident antineutrinos is obtained from Eq. (4) by the interchange $L \longrightarrow R$. An interesting result ensues for $\mathrm{v} \rightarrow 0$ : 


$$
\lim _{\mathrm{v} \rightarrow 0} \frac{\frac{1}{\mathrm{~N}} \frac{\mathrm{dN}}{\mathrm{dv}}(\bar{\nu})}{\frac{1}{\mathrm{~N}} \frac{\mathrm{dN}}{\mathrm{dv}}(\nu)}=\frac{\sigma_{\operatorname{tot}^{(\nu N)}}}{\sigma_{\operatorname{tot}^{(\nu N)}}(\overline{\mathrm{N}})}+0\left(\frac{1}{\log \frac{1}{\mathrm{~V}}}\right)
$$

This is, of course, a consequence of the fact that the denominator in Eq. (4) is proportional to $v_{\text {tot }}$. The approach to the limit, however, appears to be slow, and it may be beller to use moments of the $\mathrm{v}$ distribution. As discussed by Paschos and Zakharov ${ }^{3}$, these may be valuable in their own right, as they are not without theoretical interest. The moments of special interest are

$$
\left\langle v^{n}\right\rangle=\frac{1}{N} \int_{0}^{1} d v v^{n} \frac{d N}{d v}
$$

where we need not restrict oursclves to integral or even positive n. Assuming scaling, and returning to Eq. (5), wo may easily compute the moments defined above. For a neutrino incident, we have

$$
\left\langle v^{n}\right\rangle=\frac{\int_{0}^{1} d x x^{n} F_{2}(x)\left[\frac{L}{n+1}+\frac{S}{(n+1)(n+2)}+\frac{2 R}{(n+1)(n+2)(n+3)}\right]}{\int_{0}^{1} d x F_{2}(x)\left[L+\frac{1}{2} s+\frac{1}{3} R\right]} .
$$

For an antineutrino incident on a light nucleus we again interchange $L$ and $R$. Now upon taking the ratio of $\left\langle v^{n}\right\rangle$ for antineutrinos to that for neutrinos, and letting $n \rightarrow-1$, we find

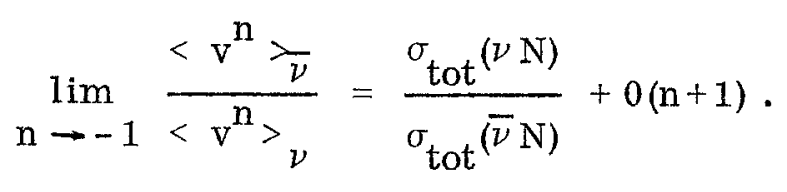


Notice that this result again (as with Eq. (10)) does not require determination of the relative flux of neutrinos or antineutrinos.

\section{FIGURE CAPTIONS}

Fig. 1 Plot of the normalized $v$ distribution as a function of $v$ using $\mathrm{F}_{2}$ (x) from electroproduction. The dashed curve shows the effect of an intermediate-boson exchange with $\mathrm{m}_{\mathrm{W}}=5 \mathrm{GeV}$ and $<\mathrm{s}>=60 \mathrm{GeV}^{2}$.

Fig. 2 Plot of the ratio of the normalized $v$ distribution given by Eq. (8) to the $\mathrm{v}$ distribution in the scaling limit. 


\section{REFERENCES}

1. G. Myatt and D. H. Perkins, Phys. Letters 34B, 542 (1971).

2. D. Cline and E. Paschos, "Testing the Adler and Gross-Llewellyn Smith Sum Rules in High Energy Neutrino Reactions," NAL-Wisconsin preprint (1973), and to be published in Phys. Rev.

3. E. A. Paschos and V. I. Zakharov, NAL preprint 100 , to be published in Phys. Rev.

4. J. D. Bjorken, Phys. Rev. 163, 1767 (1967).

5. J. D. Bjorken and E. Paschos, Phys. Rev. 185, 1975 (1969).

6. Preliminary results of the Gargamelle Collaboration, presented by P. Heusse at the XVI International Conference on High Energy Physics, Chicago-Batavia (1972); D. J. Perkins, Proceedings of the XVI International Conference on High Energy Physics, Chicago-Batavia (1972).

7. E. Paschos, Invited talk at the New York American Physical Society Meeting (1973) and NAL preprint (1973).

8. G. Miller, et al. , Phys. Rev. D5, 528 (1972). We use an empirical fit by V. Barger (private communication) to the data presented in the thesis of A. Bodek, MIT thesis, COO-3069-116 (1972). 


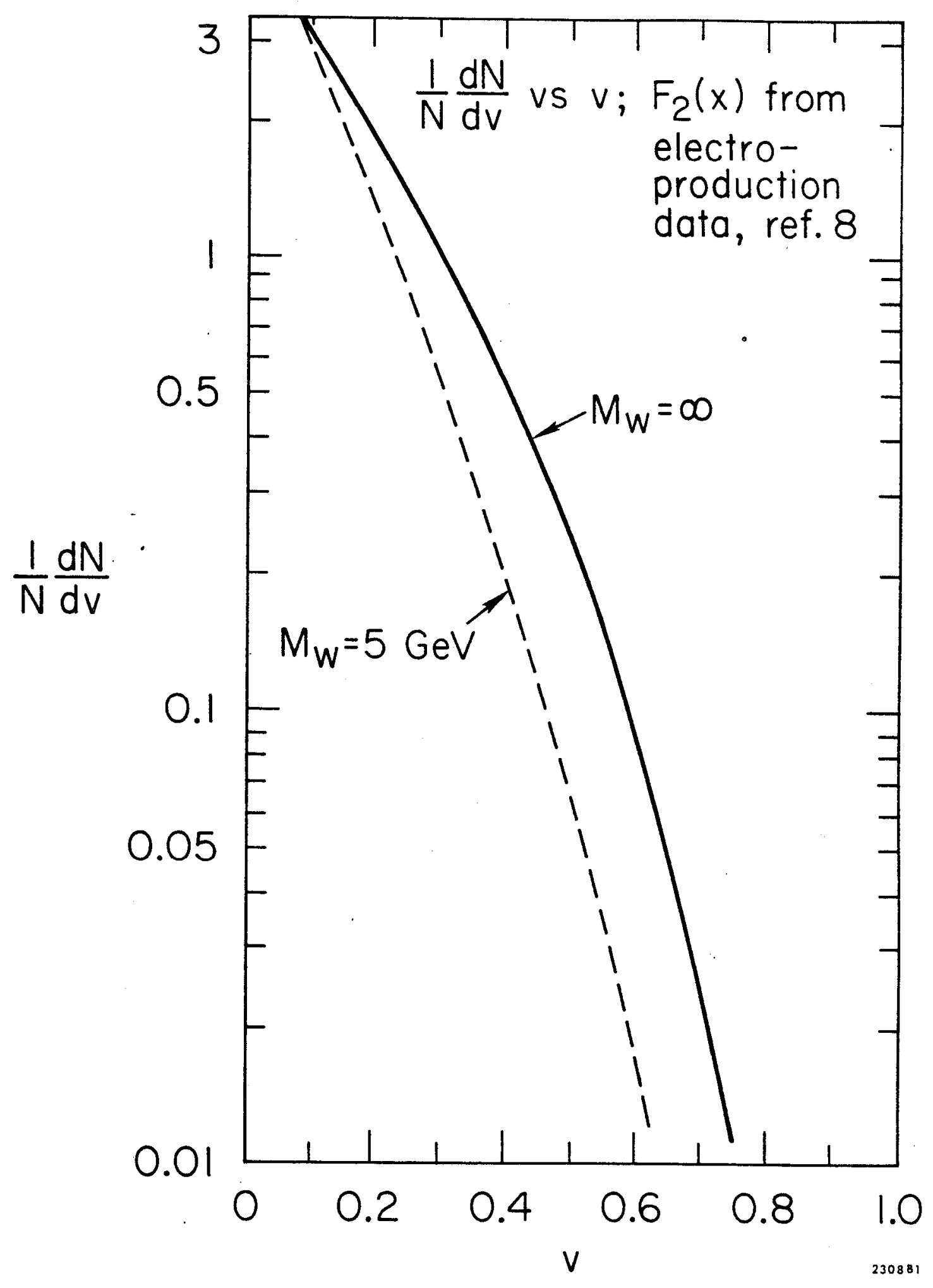

FIG. 1 


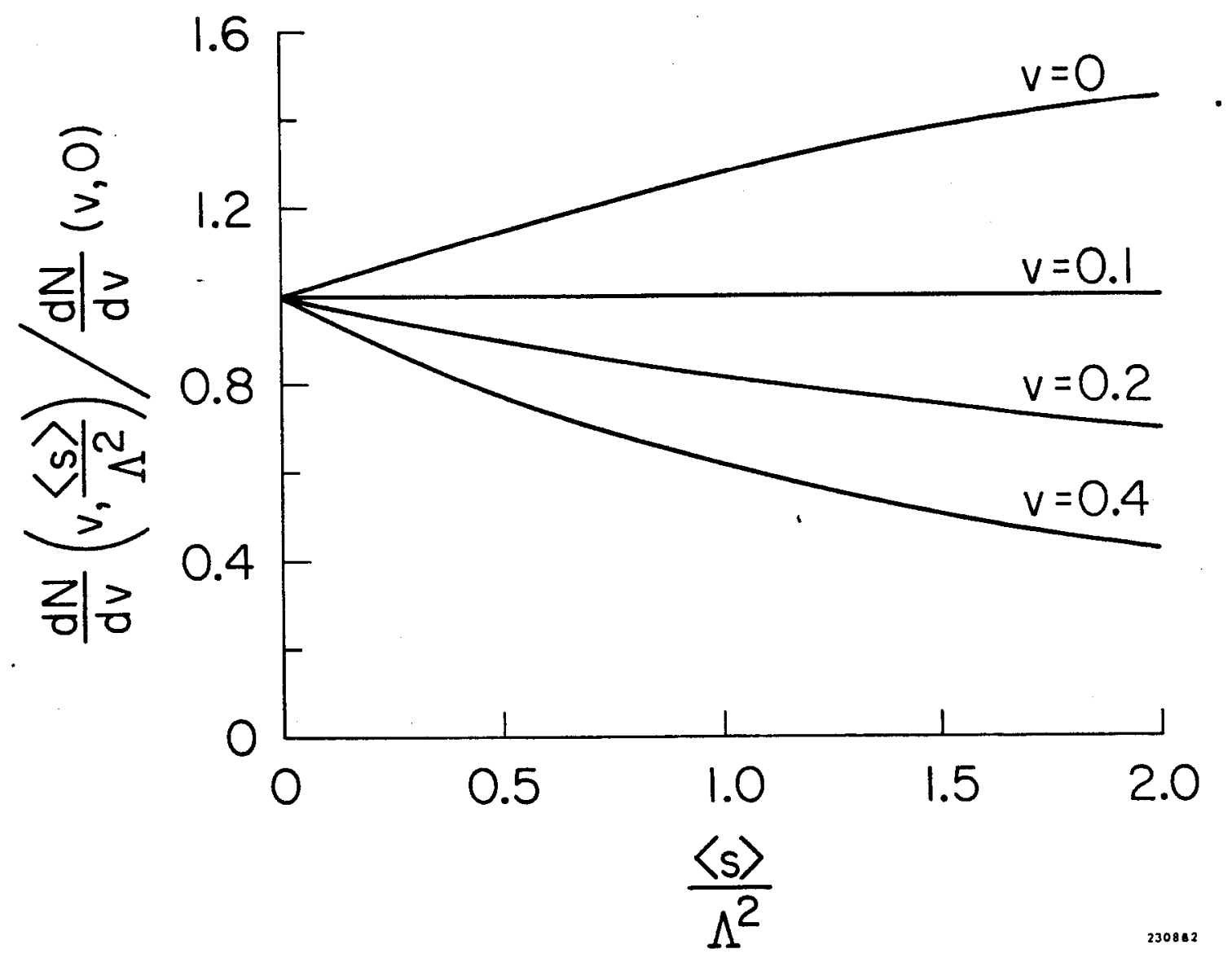

FIG. 2 